\title{
Fatigue tolerant design of rolling bearings
}

\author{
Simon P.A. Gill ${ }^{1 *}$ and Michael W.J. Lewis ${ }^{2}$ \\ ${ }^{1}$ Department of Engineering, University of Leicester, \\ University Road, Leicester, UK, LE1 7RH \\ ${ }^{2}$ Formerly of the National Centre of Tribology, ESR Technology Ltd, Whittle House, \\ 410 The Quadrant, Birchwood Park, Warrington WA3 6FW, UK.
}

\begin{abstract}
A quantitative method for assessing the influence of steel cleanliness on the fatigue life of rolling bearing raceways is presented. The approach systematically accounts for the effect of the highly variable stress state within raceways. Finite element analysis is used to determine the stress state in the bearings. A fracture mechanics model for the safe stress amplitude as a function of inclusion size is employed from Lewis and Tomkins, J Engng Tribology, 226(5) 389 (2012). The size and number of large inclusions in a large volume of steel are estimated by the Generalised Pareto Distribution. These three elements are combined to determine the failure probability of the raceway in an example rolling bearing. A sensitivity analysis to the various microstructural input parameters is conducted. It is found that the size distribution of the larger inclusions is the most important factor in controlling the fatigue resistance of rolling bearings, and that residual stresses must be considered to produce realistic predictions.
\end{abstract}

Keywords: fatigue; rolling bearings; statistics of extreme values; failure; steel cleanliness; generalised Pareto distribution. 


\section{Nomenclature}

$a \quad$ inclusion radius

$a_{0} \& a_{1}$ minimum and maximum sizes of potentially damaging inclusions

$c \& d \quad$ major and minor semi-axes of the elliptical contact area

$c_{0} \quad$ Lundberg-Palmgren index

$f(a)$ volume fraction of raceway containing particles of size greater than $a$

$f_{F} \quad$ volume fraction of the raceway containing inclusions that will lead to fatigue failure

$h \quad$ Lundberg-Palmgren depth-weighting index.

$l \quad$ raceway length

n Generalised Pareto Distribution exponent

$p_{\max }$ maximum contact pressure between the two contacting surfaces

$Z \quad$ stress weighted average depth

$z_{0} \quad$ depth of the maximum orthogonal shear stress

A cross-sectional area of the raceway

$A_{\text {con }}$ total contact area

$C_{E} \quad$ a measure of the contacting bodies net compliance

$E_{1} \& E_{2}$ Young's moduli of the ball and raceway

HV Vickers hardness of the raceway

$K_{D} \quad$ a measure of the contacting bodies radii

$L_{10} \quad$ basic rating life

$N^{e} \quad$ is the number of cycles survived raised to the power of the Weibull index

$N_{F} \quad$ average number of damaging inclusions in the raceway

$N_{P} \quad$ total number of potentially damaging inclusions in the raceway

$P \quad$ applied load

$P(a)$ probability of an inclusion of size greater than $a$

$P_{F} \quad$ probability of a bearing failing due to damaging inclusions

$P_{u} \quad$ fatigue limit load 
$R \quad$ maximum/minimum stress ratio

$R_{1} \& R_{1}^{\prime}$ radii of the ball

$R_{2} \& R_{2}^{\prime}$ radii of the raceway

$S_{S} \quad$ percentage of a population surviving

$\Delta S \quad$ survivial probability of a volume $\Delta V$

$S_{\text {limit }}$ limit stress (in $\left.\mathrm{MPa}\right)$

$V_{0} \quad$ total volume of the raceway

$\lambda_{0} \quad$ number of inclusions per unit volume in the material

$v_{1} \& v_{2}$ Poisson's ratios of the ball and raceway

$\phi(a)$ probability density of inclusions of size $a$

$\sigma_{e} \quad$ von Mises effective stress

$\sigma_{\text {res }}$ residual stress in the surface of the raceway $\tau$ shear stress amplitude exceeding the fatigue limit

$\tau_{0} \quad$ is the maximum orthogonal shear stress

$\tau_{u} \quad$ shear stress fatigue limit 


\section{Introduction}

Lifing methods for rolling element fatigue were developed by Lundberg and Palmgren in the 1940s and 50 s by analysis of many hundreds of tests on small bearings at contact stresses well in excess of $2500 \mathrm{MPa}[1,2]$. Under these conditions, significant cyclic strain-induced metallurgical changes occur [3]. These changes consist of the formation of white etching areas (WEAs) and dark etching areas (DEAs) close to the running surface that are orientated at specific angles.

It was also found that significant compressive residual stresses are developed with protracted running at these elevated stresses. However, it is difficult to state whether this would be beneficial to life under stresses less than 2500MPa without proof from testing.

In view of the distribution in time to appearance of the first spall, Lundberg and Palmgren expressed bearing fatigue in terms of L10 life, that is, the life that $90 \%$ of bearings would achieve under nominally identical loading and lubrication regimes.

However, at more modest contact stresses that are typical of industrial bearings (1200 to $1800 \mathrm{MPa}$ ), no such metallurgical changes are seen, which leads to the question whether Lundberg and Palmgren's methodology would equally apply.

Sub-surface fatigue crack initiation predominantly takes place upon non-metallic inclusions that are inevitably formed during the steel making process. Whilst modern bearing steels are significantly cleaner than those tested by Lundberg and Palmgren, crack initiation can still take place on inclusions, should they be located in the stressed contact zone.

It has been mainly assumed that the largest inclusion in a bearing raceway will be the one that is the cause of sub-surface initiated fatigue failure. However, this does not take into account the probability that this inclusion is in the most highly stressed area. It is possible that smaller but more commonly found particles could be the source of fatigue initiation, as they will have a more significant chance of being in the high stress region. Hence it is necessary to combine knowledge of the stress state in the component with an understanding of the distribution of particle sizes within the steel, the latter depending upon the steel cleanliness. This is particularly important in rolling bearings, where only a small volume of the raceway experiences very high stresses, but a much larger region experiences intermediate, but still potentially damaging, levels of stress.

The assessment of a bearing's resistance to fatigue requires the input of three factors. Firstly, an analysis of the stress state in the body is required. This is where the geometry and loading conditions are incorporated into the model, as described in section 3 . The other two factors describe the material and its response to fluctuating loads. So secondly, a model for the fatigue properties of the material, particularly the fatigue limit stress as a function of inclusion size, is required. This is discussed in section 4. Thirdly, a statistical model for the distribution of inclusions throughout the material as a function of their size must also be supplied.

This paper demonstrates this process for a rolling bearing of a given geometry. The output of this process is the probability of eventual fatigue failure of the bearing raceway as a function of the loading conditions. A sensitivity analysis is performed in section 6 to determine which of the 
required input parameters is most important in determining the fatigue tolerance of the prescribed design. 


\section{Current fatigue life methods}

\section{(a) Conventional approach}

Rolling bearings are usually selected on the basis of a percentage of failures in an application within a prescribed number of revolutions. Lundberg and Palmgren $[1,2]$ formulated a theory of fatigue failure based upon Weibull probability [4] and thus proposed a method to calculate rolling bearing life. Their life prediction model was based upon the equation

$$
\ln \left(\frac{1}{S_{S}}\right) \approx N^{e}\left(\frac{\tau_{0}^{c_{0}}}{z_{0}^{h}}\right) c z_{0} l
$$

where $S_{S}$ is the percentage of a population surviving, $N^{e}$ is the number of cycles survived raised to the power of the Weibull index, $\tau_{0}$ is the maximum orthogonal shear stress, $c_{0}$ is the LundbergPalmgren index, $z_{0}$ is the depth of the maximum orthogonal shear stress and $h$ is the LundbergPalmgren depth-weighting index. The stressed volume $V$ is given by $a z_{0} l$, where $a$ is the contact major semi-axis and $l$ is the raceway length.

Substituting for the applied bearing loads, the following equation was obtained for the basic rating life

$$
L_{10}=\left(\frac{C}{P}\right)^{p}
$$

where $p$ is an exponent ( $=3$ for ball bearings and $10 / 3$ for roller bearings). It can be seen that eqn (2) makes no provision for material structure or cleanliness, depending solely upon the basic dynamic rating $C$ and the equivalent load $P$.

This equation was adopted by ANSI/AFBMA in 1978 and by ISO in 1990 [5]. Revisions to ANSI/AFBMA Std-9 and Std-11 in 1990 [6, 7], as well as Ref 5, included factors $a_{1}, a_{2}$ and $a_{3}$ to account for different levels of reliability, material fatigue properties and lubrication quality. Factors $a_{2}$ and $a_{3}$ are usually combined to give a factor $a_{23}$ that is a function of materials and lubrication through the parameter $\kappa=v / v_{1}$, the ratio of actual to required viscosity. It is limited to a maximum value of 2.5 .

Using the basic rating life methodology of Lundberg and Palmgren, the Society of Tribologists and Lubrication Engineers (STLE) have adopted different independent factors, as summarised by Zaretsky [8]. These factors cover bearing material, melting practice, metalworking, oil filtration level and misalignment, amongst others.

\section{(b) Fatigue limit approach}

As a development of bearing life methodology, loannides and Harris [9] proposed a fatigue strength approach, similar to that in structural fatigue. Thus a fatigue limit was introduced according to the following equation by means of a shear stress fatigue limit $\tau_{u}$.

$$
\ln \left(\frac{1}{\Delta S}\right) \approx \frac{N^{e}\left(\tau-\tau_{u}\right)^{c}}{z^{h}} \Delta V
$$


where $\Delta S$ is the survivial probability of a volume $\Delta V, \tau$ is the shear stress amplitude exceeding the fatigue limit and $z$ is the stress weighted average depth. Whilst shear stress is used in eqn (3), an alternative such as von Mises stress is equally applicable. This approach suggests that there is an applied stress below which fatigue failure should not occur such that bearing life becomes infinite.

In support of the approach, loannides and Harris analysed results from rotating beam, torsional beam and reversed bending beam tests with good agreement [9]. They also carried out a small number of tests on 6309 deep groove ball bearings under two different steel qualities and lubrication conditions (high and low $\lambda$, where $\lambda$ is the ratio of oil film thickness to combined surface finish of the surfaces).

They concluded that values of the shear stress fatigue limit $\tau_{u}=300$ and 350MPa applied to the bad and good quality steels, whilst values of von Mises stress $\sigma_{u M}=519$ and 606MPa were correspondingly obtained. Applying a conversion factor of 0.55 for von Mises stress applicable to roller bearings, this equates to maximum contact stresses of 950 and $1100 \mathrm{MPa}$ respectively.

The work of loannides and Harris was extended by Harris and Barnsby [10] in which a stress-life factor $a_{S L}$ was proposed to modify the basic rating life. This factor includes, but is not limited to, fatigue endurance limit, sub- and surface-initiated failures, residual stress, hoop stress, oil film thickness, lubricant contamination and surface tractions. The stress-life factor also includes failures of rolling elements, ignored in the work of Lundberg and Palmgren.

Harris and Barnsby carried out a much more significant test programme reported in Ref [10] with a variety of bearing types up to a pitch diameter of $388.5 \mathrm{~mm}$ in materials including AISI 52100, VIMVAR M50, case-hardening and induction hardening steels. Endurance testing showed a mean von Mises fatigue limit stress of 684MPa for AISI 52100, stated to be a conservative value.

The approach adopted by Harris and Barnsby has been incorporated in the ASMELife software program [11]. Within this software it is possible to select a value for the fatigue limit stress for specific materials that reflects their metallurgy and cleanliness.

Recently, ISO 281 [12] has adopted a similar approach that considers the effects of lubricant cleanliness and viscosity as well as the concept of an endurance limit. The latter is introduced as a fatigue limit load $P_{u}$ (or $C_{u}$ ) so historically the concept of an endurance limit (or fatigue strength) has been adopted for the lifing of rolling element, so the approach to be described below is a natural development of this.

\section{Stress analysis}

(a) Finite Element model

The original application of fatigue tolerant analysis [13] utilised an analytical solution for the stress field in the component (a plate with a hole). Here the analysis is conducted within the more flexible framework of the finite element method. A rolling bearing is a good application to consider due to the very wide variety of stress histories experienced by different elements of the raceway. The geometry of a representative section of a large ball bearing is shown in Fig. 1a. Only a single ball acting on a section of the outer raceway is considered as the contact stress field between the ball 
and the raceway is very localised. The convex radius of the ball is $31.75 \mathrm{~mm}$, the concave track radius is $33.66 \mathrm{~mm}$, such that the osculation is 1.06 (or $53 \%$ ), and the concave outer race radius is $910 \mathrm{~mm}$.

The exact cross-sectional dimensions of the raceway should not be important, as long as the contact stress field is not significantly affected by the proximity of the external boundaries. The elastic properties of the ball and the raceway are assumed to be the same, with a Young's modulus of 210 GPa and a Poisson's ratio of 0.3. Exploiting the symmetry of the problem, only a quarter of the geometry needs to be modelled, as shown in Fig. 1b. A suitable range of uniformly distributed displacements are applied normal to the flat, horizontal surface of the truncated ball to press the ball into the raceway. The total load is subsequently calculated from the reaction force on this surface, and is typically in the range of $10-80 \mathrm{kN}$.

The finite element mesh is refined near the contact in an area defined by an ellipsoid. Due to the curvature of the raceway, the contact zone is not axisymmetric, with the contact size across the raceway being much larger than the contact size in the rolling direction. The variation in the von Mises stress within the ball and the raceway is shown in Fig. 1b for a large applied load. The maximum von Mises stress occurs a small distance below the centre of the contact.

(a) Fatigue tolerance method

The output required from this stress analysis for the evaluation of the fatigue tolerance of the bearing outer raceway is determined as follows. As the ball moves around the raceway, any crosssection of the raceway will experience the same fluctuating stress state as the ball passes over it. The maximum stress amplitude of this fluctuation will occur when the centre of the ball is within the cross-sectional plane, as shown in Fig. 1b. Firstly, we identify the cross-sectional area of the raceway as the area $A$, as shown in Fig. 1 a. The total volume of the raceway $V_{0}$ can then be determined by sweeping this area through $360^{\circ}$ about the centre of the bearing such that

$$
V_{0}=2 \pi \int_{A} r d A
$$

where $r$ is the distance from the centre of the bearing to a point within the outer raceway crosssection. The objective of the stress analysis is to determine the fraction of this volume that has a von Mises effective stress $\sigma_{e}$ that is above a given stress $S$. The logical expression

$$
\sigma_{e}>S= \begin{cases}1 & \text { if true } \\ 0 & \text { if false }\end{cases}
$$

is used to determine the swept volume in which the condition $\sigma_{e}>S$ is satisfied

$$
V(S)=2 \pi \int_{A}\left(\sigma_{e}>S\right) r d A .
$$

This is then normalised to define the volume fraction of the raceway in which $\sigma_{e}>S$

$$
f(S)=\frac{V(S)}{V_{0}}
$$

This function is plotted in Fig. 2a for the range of applied loads considered, from $1.6 \mathrm{kN}$ up to $80 \mathrm{kN}$. The curves become increasingly smooth as the load rises as the stress field extends over more finite 
elements in the simulation and is more accurately represented by the discretised field. As one would expect, the volume fraction above a certain stress level increases as the applied load is increased.

\section{(b) Comparison with analytical solution}

An analytical solution for the problem of two, doubly curved surfaces in contact is given in Roark's Formulas for Stress and Strain [14] and is widely used in the assessment of bearings. Therefore it is of interest to see how this compares with the results of the finite element solution. For small deformations, the analytical solution states that the shape of the elliptical contact area is given by

$$
c=\alpha\left(P K_{D} C_{E}\right)^{\frac{1}{3}} \quad d=\beta\left(P K_{D} C_{E}\right)^{\frac{1}{3}}
$$

where $c$ and $d$ are the major and minor semi-axes of the elliptical contact area, such that the total contact area is given by $A_{c o n}=\pi c d$, and $P$ is the applied load, $K_{D}=\frac{1.5}{\frac{1}{R_{1}}+\frac{1}{R_{2}}+\frac{1}{R_{1}^{\prime}}+\frac{1}{R_{2}^{\prime}}}$ is a measure of the contacting bodies radii, and $C_{E}=\frac{1-v_{1}^{2}}{E_{1}}+\frac{1-v_{2}^{2}}{E_{2}}$ is a measure of their compliance. Here $E_{1}=E_{2}=$ $210 \mathrm{GPa}$ and $v_{1}=v_{2}=0.3$. The two convex (positive) radii of the ball are $R_{1}=R_{1}^{\prime}=31.75 \mathrm{~mm}$ and the two concave (negative) radii of the raceway are $R_{2}=-1.06 R_{1}$ and $R_{2}^{\prime}=-910 \mathrm{~mm}$. The two dimensionless constants can then be interpolated from a table of values defined by the value of $K_{D}$ such that $\alpha=3.00$ and $\beta=0.46$ [14]. The maximum contact pressure between the two surfaces is given by

$$
p_{\max }=\frac{1.5 P}{A_{\text {con }}}=\eta P^{\frac{1}{3}}
$$

where $\eta$ is a geometrical constant defined by (8). The stresses in the raceway are expected to scale with the contact pressure so that the von Mises effective stress $\sigma_{e}$ is also proportional to $P^{\frac{1}{3}}$. The size of the stress contours in the cross-section (see Fig. 1 b) will scale with the dimensions of the contact radius. Thus the width and height of the stress field will scale as $P^{\frac{1}{3}}$, and hence the area contained by a particular stress contour will scale as $P^{\frac{2}{3}}$.

To compare the simulation results with the above solution, Fig. $2 \mathrm{a}$ is re-plotted in Fig. $2 \mathrm{~b}$ with the axes renormalized such that, if the analytical solution is always valid, the curves should all collapse onto the same master curve. The renormalizations are such that the measure of stress becomes $\frac{s}{p_{\max }}$, and the measure of volume fraction becomes $\frac{f(S)}{P^{\frac{2}{3}}}$. The particular value of the volume fraction is only relative, and depends on the size of the raceway cross-section adopted. Curves are only plotted for four selected loads for clarity. The curves for intermediate loads lie proportionally between those shown. It is clear from Fig. $2 b$ that the curves do not exactly collapse on to a single master curve, although the general scaling is roughly applicable. This is because the analytical solution is only valid for small contacts. It is noted that for similar calculations by the authors of contact between an identical ball and a flat surface under the same range of loads, the curves do collapse much more precisely onto the same curve.

The relationship between the maximum contact pressure and the maximum von Mises stress is given to be $\frac{s_{\max }}{p_{\max }}=0.557$ for a cylinder contacting a flat substrate [15]. The constant of 
proportionality is not easily defined for the general case of two doubly curved surfaces in contact, but it is found that it is about 0.65 for a load of $32 \mathrm{kN}$, and that it increases steadily with the applied load, reaching a value of 0.68 at $80 \mathrm{kN}$. This represents a significant difference of around $20 \%$ from the cylinder case and hence shows that it is not a simple matter of employing a single master curve for all contact problems. Values of about 0.63 are cited in the literature.

As one might expect, the stress field varies in shape as well as magnitude as the geometry changes. The increase in the ratio of von Mises to contact stress with load is anticipated to be due to effects of large deformations. With a small osculation, typical in bearing applications, the contact area between the ball and the raceway expands quickly with the applied load. The contact pressure between the two objects is normal to the surface. For small loads, this is almost entirely in the (vertical) direction of the applied load, but as the contact area enlarges, the horizontal component becomes more significant, as the ball starts to touch the side walls of the raceway.

\section{(c) Effect of residual stress}

The raceways of some rolling bearings are surface hardened, typically by induction hardening or carburising. This is often used to gain high surface hardness whilst maintaining a tougher core. This process induces a compressive residual stress in the near-surface of the component, which is generally found to greatly improve its fatigue resistance. Therefore, it is necessary to consider the contribution from residual stresses to see whether results are comparable to service experience.

Exact details about the residual stress field are unknown, but it is expected to be compressive, reasonably uniform to a certain distance below the treated surface, and parallel to the plane of this surface. Further away from the surface the residual stress must be tensile in order to ensure overall force equilibrium. Fig. 3a shows the variation in the von Mises stress field in the cross-section beneath the ball centre. Here it is assumed that a uniform compressive residual stress field exists to a depth sufficient to cover the entirety of the high stress region, and that the orientation of the surface above this region is sufficiently close to the horizontal ( $x-y$ plane) that it can be assumed to act entirely within this plane. Therefore, in the absence of specific detailed information, a simple representation of the residual stress field is to replace the stress components in the horizontal plane $\sigma_{x}$ and $\sigma_{z}$ by $\left(\sigma_{x}-\sigma_{\text {res }}\right)$ and $\left(\sigma_{z}-\sigma_{\text {res }}\right)$, where $\sigma_{\text {res }}=400 \mathrm{MPa}$ is taken to be the magnitude of the (compressive) near-surface residual stress field. The von Mises stress in the presence of the residual stress is then defined as

$$
\sigma_{e}^{r e s}=\sqrt{\frac{1}{2}\left[\left(\sigma_{x}-\sigma_{z}\right)^{2}+\left(\sigma_{x}-\sigma_{y}-\sigma_{r e s}\right)^{2}+\left(\sigma_{z}-\sigma_{y}-\sigma_{r e s}\right)^{2}+6\left(\tau_{x y}^{2}+\tau_{x z}^{2}+\tau_{y z}^{2}\right)\right]}
$$

Fig. $3 \mathrm{~b}$ shows the difference between the stress field in the presence of the residual stress, calculated using (10), and the original stress field, shown in Fig. 3a. The residual stress is applied everywhere, such that the unstressed (blue) regions in Fig.3a now have an increased stress level of $400 \mathrm{MPa}$. This is unphysical but irrelevant, as the increase in the stress in these regions is still much too small to initiate fatigue and hence they do not play any part in the overall calculation. The relevant observation in Fig. $3 \mathrm{~b}$ is that the area where the von Mises stress is reduced by the value of the applied residual stress, $400 \mathrm{MPa}$, completely covers the high stress area in Fig. 3a. This is where fatigue will be initiated. Plotting the equivalent stress curves of Fig.2a show that the addition of the residual stress field effectively just translates the high stress portion of the stress curve to the left, 
such that a good approximation of the consequences of the residual stress field are obtained by simply replacing $S$ by $\left(S-\sigma_{\text {res }}\right)$ on the horizontal axis. This is a simplified model, but introducing a more sophisticated model will not have an observable qualitative effect on the final results.

\section{Fatigue properties of the material}

Having characterised the fluctuating stress state in the raceway, it is now necessary to relate the state of stress in the volume to its propensity for initiating fatigue. It is assumed that fatigue initiates at inclusions in the steel. Lewis and Tomkins [16] have proposed (based upon the work of Murakami [17]) the following relationship between the size of an inclusion and the limit stress amplitude for eventual fatigue failure

$$
S_{\text {limit }}(a)=1.63 \frac{(H V+120)\left(\frac{1-R}{2}\right)^{\alpha}}{\left(0.49 \pi a^{2}\right)^{\frac{1}{12}}}
$$

where the limit stress is given in MPa, $H V=750$ is the Vickers hardness of the steel, $R=-1$ is the $\mathrm{max} / \mathrm{min}$ stress ratio and $\alpha=0.226+H V \times 10^{-4}$. The inclusions are assumed to be ellipsoidal with semi-axes $a$ and $b$ with $b / a=0.49$ such that the cross-sectional area of the particles is $\pi a b$ or $0.49 \pi a^{2}$. Equation (11) is plotted in Fig. 4 . If the stress amplitude is below the line then no fatigue will be initiated. If the stress amplitude is above the line, then fatigue failure will occur eventually.

It is now possible to re-map the horizontal stress axis in Fig. $2 \mathrm{~b}$ using (11), $S(a)$, to calculate the volume fraction above the limit stress, $f(a)$, as a function of the inclusion size. Fig. 5 shows the volume fraction of the raceway that experiences stress amplitudes sufficiently large to initiate fatigue in particles of size $a$ and above for different applied loads. Here the size of the ellipsoidal particles is defined to be the largest radius which is roughly equivalent to the smallest diameter for the assumed aspect ratio. As the load increases, larger proportions of the raceway volume experience stresses which will activate a greater range of particles sizes.

\section{Microstructural description}

Figure 5 defines the fraction of the raceway in which an inclusion of a particular size has the potential to act as a site for the initiation of fatigue failure. It is now necessary to combine this with a description of the particle size distribution in the steel to determine the probability of finding an inclusion of a potentially damaging size in a sufficiently stressed region of the raceway. It is not possible to determine the probability of finding such large inclusions using a brute force approach, as the large number of micrographics samples that would need to be examined to have confidence that statistically significant numbers of such particles had been detected would be beyond practical analysis. The size distribution is therefore usually interpreted from a smaller, more manageable, number of micrographs using the statistics of extreme values [18-21]. There are a number of different approaches to this problem [18]. In this paper we adopt the Generalised Pareto Distribution (GPD) $[13,18]$, where here it is expressed in the following form

$$
P(a)=\left(\frac{a_{1}-a}{a_{1}-a_{0}}\right)^{n}
$$


This form assumes that there is always a maximum size for any inclusions that can ever be found in a sample, $a_{1}$. It also assumes that inclusions below a specific threshold size, $a_{0}$, will never be potentially damaging and do not need to be considered in the analysis. Therefore we are interested in particles of size $a_{0} \leq a \leq a_{1}$. The exponent $n$ is the third fitting parameter for the distribution. The probability $P(a)$ is therefore the cumulative probability of finding inclusions of a potentially damaging size, $a$. Typical values for the threshold and maximum inclusion sizes are $a_{0}=5$ microns and $a_{1}=30$ microns. The distribution function (12) is plotted in Fig. 6a for a range of exponent values. The meaning of these distributions is often more easily interpreted from the probability density function (PDF)

$$
\phi(a)=-\frac{d P}{d a}=\frac{n}{a_{1}-a_{0}}\left(\frac{a_{1}-a}{a_{1}-a_{0}}\right)^{n-1}
$$

which defines the frequency of occurrence of inclusions of a particular size. The PDFs for the distributions illustrated in Fig. 6a are shown in Fig. 6b. They demonstrate that a uniform distribution of particles sizes is expected for $n=1$, i.e. there is equal chance of finding particles of any size in the considered range, whilst for $n<1$ there is a greater chance of finding large particles than small ones. This is not anticipated to be the case, and therefore one requirement on the exponent is that $n>1$. As the value of $n$ increases, the probability of large inclusions occurring becomes more and more unlikely. The value of $n=11$ is typical of the values previously considered [13].

\section{Probability of failure - a sensitivity analysis}

The stress distribution in the raceway has been combined with the fatigue properties of the material, and is summarised in Fig. 5. This is now amalgamated with the microstructural picture of the inclusions in the material provided by Fig. $6 \mathrm{~b}$ to determine the overall probability of failure of these components. Firstly, it is necessary to define the number of potentially damaging inclusions, with sizes $a_{0} \leq a \leq a_{1}$, that are estimated to occur in the body of the raceway. Hence we let $\lambda_{0}$ be the number density of these inclusions in the steel under consideration. Given that we have determined the total volume of the raceway, $\mathrm{V}_{0}$, in (4), the total number of potentially damaging inclusions in the raceway is $\mathrm{N}_{\mathrm{P}}=\lambda_{0} \mathrm{~V}_{0}$. However, these inclusions can only actually lead to the initiation of fatigue damage if they are in a region of the raceway that experiences a sufficiently high enough stress to activate it. Combining Fig. 5 and Fig. 6b, the volume fraction of the raceway containing actually damaging inclusions that will lead to fatigue failure is

$$
f_{F}=\int_{a_{0}}^{a_{1}} f(a) \phi(a) d a .
$$

This is the sum over the product of the volume fraction of the raceway, $f(a)$, containing particles which experience stress amplitudes above the stress limit (11), and the probability that inclusions of this critical size exist, $\phi(a)$. The average number of actually damaging inclusions that might be expected to be found in the raceway is therefore $N_{F}=N_{P} f_{F}$. The chances of finding a particular number of damaging inclusions in a body obeys the Poisson distribution, which gives the probability of failure for an event that occurs on average with a frequency $N_{F}$ such that

$$
P_{F}=1-\exp \left(-N_{F}\right)
$$


The predictive capacity of this expression is now investigated. The first factor to be considered is the effect of a residual stress. This is then followed by a study of the sensitivity of this rolling bearing to the four microstructural parameters: $a_{0}, a_{1}, n$ and $\lambda_{0}$. In all studies, the volume of the raceway is $V_{0}=0.00454 \mathrm{~m}^{3}$. Apart from the final study, we assume that $\lambda_{0}=1$ inclusion per $\mathrm{mm}^{3}$ such that the total number of potentially damaging inclusions is $N_{P}=4,540,000$. For the geometry considered, in (9) $\eta=5.95 \times 10^{7} \mathrm{~N}^{\frac{2}{3}} / \mathrm{m}^{2}$, such that the maximum contact pressures are $1.28,1.61$ and $1.85 \mathrm{GPa}$ at applied loads of 10,20 and $30 \mathrm{kN}$ respectively.

(a) Residual stress, $\sigma_{\text {res }}$

Fig. 7 shows the failure probability (15) as a function of the load applied to the ball for the case where a compressive $400 \mathrm{MPa}$ residual stress is superimposed on the raceway stress state, and the case where no residual stress is considered. It is clear that including the residual stress is very important, and that it has a huge effect on the typical fatigue failure load of the bearing. Fig.7 indicates that one bearing in a thousand will fail by fatigue if the applied load is $12 \mathrm{kN}$ without a residual stress. The allowable applied load for this failure rate is increased to $31 \mathrm{kN}$ if the $400 \mathrm{MPa}$ residual stress is included. This is a reasonable value for the failure load of bearings of this type, and therefore the effects of the residual stress are included in all the subsequent analyses.

(b) The exponent $n$

Three different values for the exponent $n$ are chosen and the results are plotted in Fig. 8. The smaller the exponent, the greater the frequency of large inclusions in the size distribution. The failure probability shows that for the lowest value, $n=2$, a raceway is predicted to suffer from fatigue failure as soon as the applied load reaches the critical value at which only the largest inclusions will cause eventual failure. This is because the probability of finding such inclusions is reasonably large. This probability decreases significantly as $n$ is increased. An increase to $n=5$ only makes a noticeable difference in the onset of fatigue failure occurring if a failure probability for the bearing of one in ten or above is allowable. The largest value considered, $n=11$, is used in [18] and demonstrates that the fatigue life of the component can be significantly increased for a given applied load if the number of large inclusions can be made sufficiently infrequent. Even though large inclusions still occur, it is then unlikely that they will be found in the small highly stressed region of the raceway. For $n=11$ the probability of finding an inclusion of size greater than 21 microns is one in a hundred thousand. Hence $n=11$ is considered to represent an extremely clean steel.

(c) Maximum inclusion size, $a_{1}$ Three maximum particle sizes are considered: $a_{1}=30,50$ and 70 microns. The effect of varying the maximum inclusion size is shown for two cases in Figs. 9a and $9 \mathrm{~b}$ in which $n=2$ and $n=11$. As expected, both figures demonstrate that the failure probability increases as the maximum inclusion size in the steel is increased. As in Fig. 8, the case of $n=2$ in Fig. 9a illustrates a sharp transition from no chance of failure to $100 \%$ chance of eventual failure once the applied load reaches the limit at which the highest stress is large enough to activate the largest inclusions in the body. The similarity between $a_{1}=50$ microns and $a_{1}=70$ microns shows that the stress limit (11) is not sensitive to very large inclusion sizes. Fig. $9 \mathrm{~b}$ is likely to be more representative of the inclusion distribution in an actual clean steel. It shows a clearer differentiation between the three cases, as the chances of finding the larger inclusions in the highly stressed region becomes less likely. As in Fig.7, for $a_{1}=30$ 
microns one in a thousand raceways are predicted to suffer fatigue failure for an applied load of $31 \mathrm{kN}$. As the maximum inclusion size increases to 50 and 70 microns, this applied load decreases to $26 \mathrm{kN}$ and $24 \mathrm{kN}$ respectively. This is a significant difference in load bearing capacity, with a variation of about $25 \%$ between the two extreme cases.

\section{(d) Number of inclusions, $N_{P}$}

The number of potentially damaging inclusions in the raceway, $N_{P}=\lambda_{0} V_{0}$, can be varied by either changing the density of inclusions within the steel, $\lambda_{0}$, or the size of the raceway, $V_{0}$. Fig. 10 shows the effect of reducing the number of potentially damaging inclusions in the steel by a factor of 10 and 100. Assuming the dimensions of the raceway cannot be changed, this would be achieved in investing in a significantly cleaner, and more expensive, steel. It is clear that for a low acceptable failure probability of $10^{-3}$ the bearing is fairly insensitive to changes in this parameter, with an order of magnitude change in the number of inclusions only increasing the allowable applied load for the bearing by $1 \mathrm{kN}$. For a higher acceptable failure probability of 0.1 a larger increase of $2.5 \mathrm{kN}$ is possible. Hence it would certainly appear, for this very large rolling bearing, that the benefits of decreasing the number of inclusions in the steel are relatively small compared to the additional effort and expense involved. In addition, it is also useful to note that it is probably not normally necessary to expend extensive micrographic effort in determining the parameter $\lambda_{0}$ to a high degree of accuracy.

\section{Conclusions}

The fatigue tolerance assessment process of Yates et al [13] has been applied to the practical case of fatigue in the raceway of rolling bearings. The technique has been extended to the case of more general geometries and loading conditions through application of the finite element method. The case study of a particular rolling bearing is illustrated in some detail. It is found that finite element analysis is required for accurate simulation of doubly curved surfaces which undergo large deformations.

The final output of the model is the probability of eventual fatigue failure of the raceway as a function of the applied ball load. This depends on the material models for the dependence of the fatigue limit on inclusion size and the distribution of inclusion sizes within the steel. With a residual stress of $400 \mathrm{MPa}$ the threshold for rolling bearing fatigue failure at a probability of 0.001 are shown to be in a range of applied loads from $24-32 \mathrm{kN}$ depending on inclusion sizes and numbers. This corresponds to a range of contact pressures from 1.7 to $1.9 \mathrm{GPa}$. The sensitivity of the failure probability is investigated with respect to the input parameters. It is found that incorporation of a residual stress field is extremely important in the analysis of bearing raceways, with a compressive $400 \mathrm{MPa}$ residual stress increasing the one in a thousand failure load from $12 \mathrm{kN}$ to $31 \mathrm{kN}$. It is acknowledged that the residual stress model is very simplistic, and that further work should be done to represent this in a more realistic manner.

However, the general conclusions are not expected to change, unless the high stress region moves below the near-surface residual stress zone. As expected, decreasing the frequency of very large inclusions is beneficial to the life of the component. This is particularly true for bearing raceways, 
where only a small volume of the body experiences the highest stresses, so the inclusions found in this region have a lower chance of being large enough to initiate eventual fatigue failure. The total number of inclusions is shown to be less important, as long as the chances of encountering large inclusions is sufficiently rare. However, the primary output of this work is a quantification of the effects of rare, large inclusions, including exactly what rare and large mean in this context.

\section{References}

1. Lundberg $G$ and Palmgren A. Dynamic capacity of rolling bearings. Acta Polytechnica, Mechanical Engineering Series. Royal Swedish Academy of Engineering Sciences, Vol 1, No 3, pp 7, 1947.

2. Lundberg $G$ and Palmgren A. Dynamic capacity of roller bearings. Acta Polytechnica, Mechanical Engineering Series. Royal Swedish Academy of Engineering Sciences, Vol 2, No 4, pp 96, 1952.

3. Zwirlein $\mathrm{O}$ and Schlicht $\mathrm{H}$. Rolling contact fatigue mechanisms - accelerated testing vs field performance. Rolling Contact Fatigue Testing of Bearing Steels. ASTM STP 771 pp 358-379. 1982.

4. Weibull W. A statistical representation of fatigue failures in solids. Acta Polytechnica, Mechanical Engineering Series. Royal Swedish Academy of Engineering Sciences, Vol 5, No 9, pp 49, 1949.

5. ISO 281-1. Rolling bearings - dynamic load ratings and rating life. International Standards Organisation. 1990.

6. ANSI/AFBMA Std 9-1990. Load ratings and fatigue life for ball bearings. ANSI/AFBMA.

7. ANSI/AFBMA Std 11-1990. Load ratings and fatigue life for roller bearings. ANSI/AFBMA.

8. Zaretsky E. STLE life factors for rolling bearings. STLE. 1992.

9. Ioannides $E$ and Harris T A. A new fatigue life model for rolling bearings. ASME Jour of Tribology, Vol 107, pp 367. July 1985

10. Harris T A and Barnsby R M. Life ratings for ball and roller bearings. I Mech E Jour of Eng Tribology. Proc I Mech Eng, Vol 215, Part J, pp 577. 2001.

11. Barnsby $\mathrm{R}$ et al. Life ratings for modern rolling bearings - a design guide for the application of International Standard ISO 281/2. Trib Vol 14. 2003.

12. ISO 281. Rolling bearings - dynamic load ratings and rating life. International Standards Organisation. 2007.

13. Yates J R, Shi G, Atkinson H V, Sellars C M and Anderson C W. Fatigue tolerant design of steel components based on the size of large inclusions. Fatigue Fract Engng Mat Struct Vol 25, pp 667, 2002.

14. Young W C, Budynas R G and Sadegh A M. Roark's Formulas for Stress and Strain. McGrawHill Inc, Eighth Edition, 2012.

15. Harris T A and Kotzalas M N. Essential Concepts of Bearing Technology. CRC Press, Fifth Edition, 2006.

16. Lewis $\mathrm{M} \mathrm{W} \mathrm{J} \mathrm{and} \mathrm{Tomkins} \mathrm{B.} \mathrm{A} \mathrm{fracture} \mathrm{mechanics} \mathrm{interpretation} \mathrm{of} \mathrm{rolling} \mathrm{bearing} \mathrm{fatigue.}$ Proc IMechE Part J: J Engineering Tribology, Vol 226, pp 389, 2012. 
17. Murakami Y. Metal fatigue: effects of small defects and non-metallic inclusions. Elsevier 2002.

18. Shi G, Atkinson H V, Sellars C M and Anderson C W. Application of the generalized Pareto distribution to the estimation of the size of the maximum inclusion in clean steels. Acta Mater. Vol 47, pp 1455, 1999.

19. Shi G, Atkinson H V, Sellars C M and Anderson C W. Comparison of extreme values statistical methods for predicting maximum inclusion size in clean steels. Ironmaking Steelmaking, Vol 26, pp 23, 1999.

20. Atkinson H V, Shi G, Sellars C M and Anderson C W. Statistical prediction of inclusion sizes in clean steels. Mat. Sci. Tech., Vol 16, pp 1175, 2000.

21. Anderson C W, Shi G, Atkinson H V and Sellars C M. The precision of methods using the statistics of extremes for the estimation of the maximum size of inclusions in clean steels.

Acta Mater., Vol 48, pp 4235, 2000. 


\section{Figure captions}

Figure 1 : (a) Geometry of the roller bearing, considering a single ball on the outer raceway, and (b) stress analysis of the ball in contact with the raceway. A quarter of the problem can be analysed due to symmetry. The load is applied downwards in the y-direction on the upper flat surface. The stresses shown are von Mises stresses. The ellipsoidal region around the contact area demarcates an area of mesh refinement.

Figure 2 : Distribution of peak stress within the raceway volume. (a) The volume fraction above a given stress $S, f(S)$, as a function of the stress $S$. As the applied load $P$ increases, the volume fraction above a given stress increases. (b) Some of the profiles from (a) renormalized using analytical contact theory. The profiles do not collapse onto precisely the same master curve, indicating that the stress state under the indenter does not exactly scale with the load according to Hertzian contact theory.

Figure 3: (a) Von Mises stress profile in the raceway cross-section, $\sigma_{e}$. (b) The change in the von Mises stress, $\sigma_{e}^{r e s}-\sigma_{e}$, when a horizontal $400 \mathrm{MPa}$ compressive stress is incorporated into the von Mises stress calculation. This shows that the (red) region where the highest stresses act in (a) is completely covered by the (blue) region in (b) where the residual stress reduces the von Mises stress by approximately $400 \mathrm{MPa}$.

Figure 4: The fatigue limit stress (11) as a function of inclusion size.

Figure 5: Combining Fig. $2 \mathrm{~b}$ and Fig. 4 gives the volume fraction of the raceway, $f(a)$, where the stress is large enough to initiate fatigue from inclusions of size $a$.

Figure 6: The (a) cumulative probability GPD function (12), and (b) its probability density function (13), for a threshold size of $a_{0}=5$ microns, maximum size $a_{1}=30$ microns, and various values of the exponent $n$.

Figure 7: The failure probability for a sample with $a_{0}=5$ microns, $a_{1}=30$ microns, $n=11$, with a compressive residual stress field $(400 \mathrm{MPa})$ and without $(0 \mathrm{MPa})$.

Figure 8: The failure probability for a sample with $a_{0}=5$ microns, $a_{1}=30$ microns, a compressive residual stress field (400MPa) for various values of $n$.

Figure 9: The failure probability for a sample with a compressive residual stress field (400MPa) with $a_{0}=5$ microns and values of $a_{1}=30,50$ and 70 microns for (a) $n=2$ and (b) $n=11$.

Figure 10: The failure probability for a sample with a compressive residual stress field (400MPa) with $a_{0}=5$ microns, $a_{1}=30$ microns, $n=11$ for different values for the number of inclusions in the raceway $N_{P}$. 


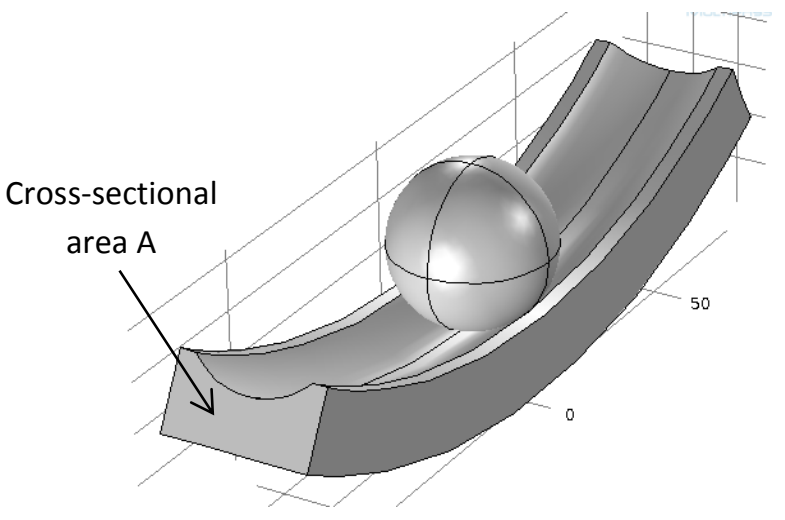

(a)

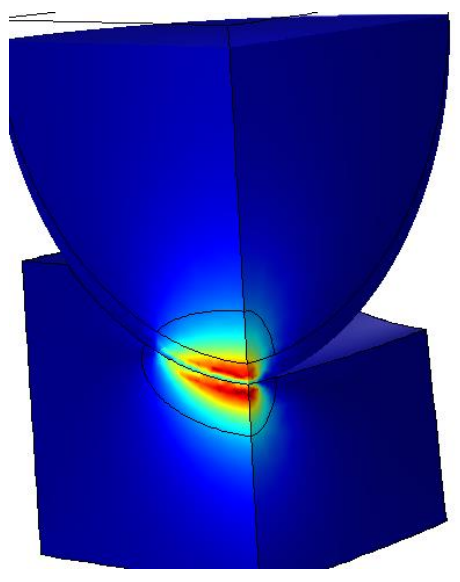

(b)

Figure 1 : (a) Geometry of the roller bearing, considering a single ball on the outer raceway, and (b) stress analysis of the ball in contact with the raceway. A quarter of the problem can be analysed due

to symmetry. The load is applied downwards in the y-direction on the upper flat surface. The stresses shown are von Mises stresses. The ellipsoidal region around the contact area demarcates an area of mesh refinement. 


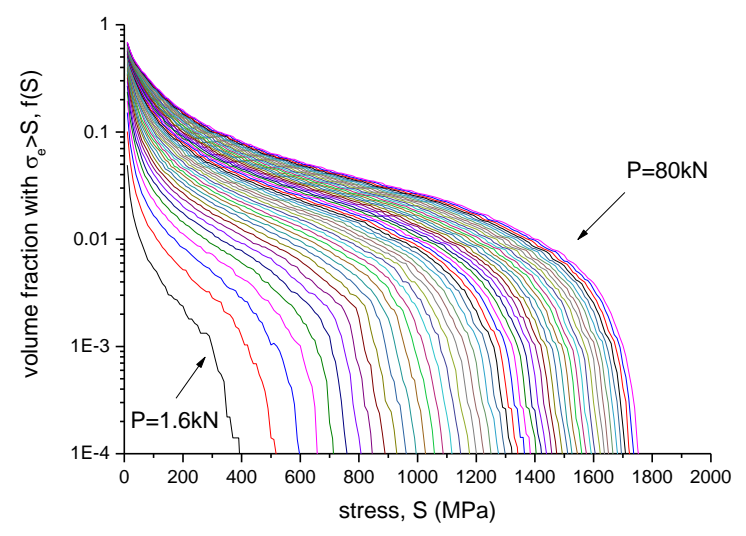

(a)

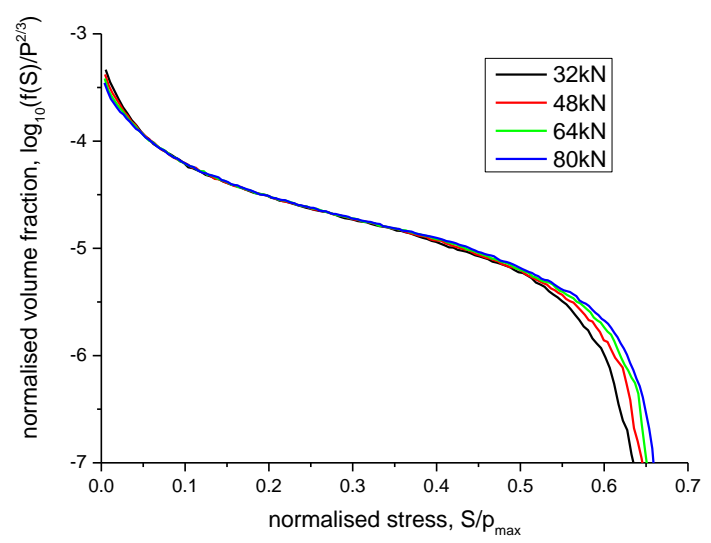

(b)

Figure 2 : Distribution of peak stress within the raceway volume. (a) The volume fraction above a given stress $S, f(S)$, as a function of the stress $S$. As the applied load $P$ increases, the volume fraction above a given stress increases. (b) Some of the profiles from (a) renormalized using analytical contact theory. The profiles do not collapse onto precisely the same master curve, indicating that the stress state under the indenter does not exactly scale with the load according to Hertzian contact theory. 


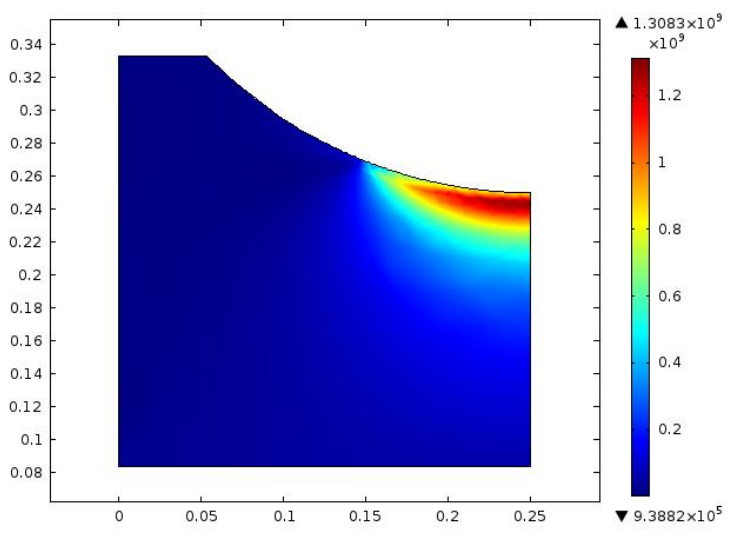

(a)

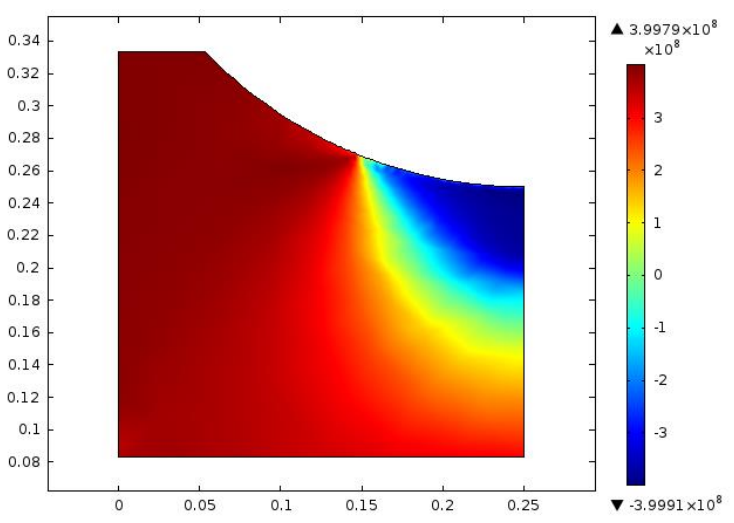

(b)

Figure 3: (a) Von Mises stress profile in the raceway cross-section, $\sigma_{e}$. (b) The change in the von Mises stress, $\sigma_{e}^{r e s}-\sigma_{e}$, when a horizontal $400 \mathrm{MPa}$ compressive stress is incorporated into the von Mises stress calculation. This shows that the (red) region where the highest stresses act in (a) is completely covered by the (blue) region in (b) where the residual stress reduces the von Mises stress by approximately 400MPa. 


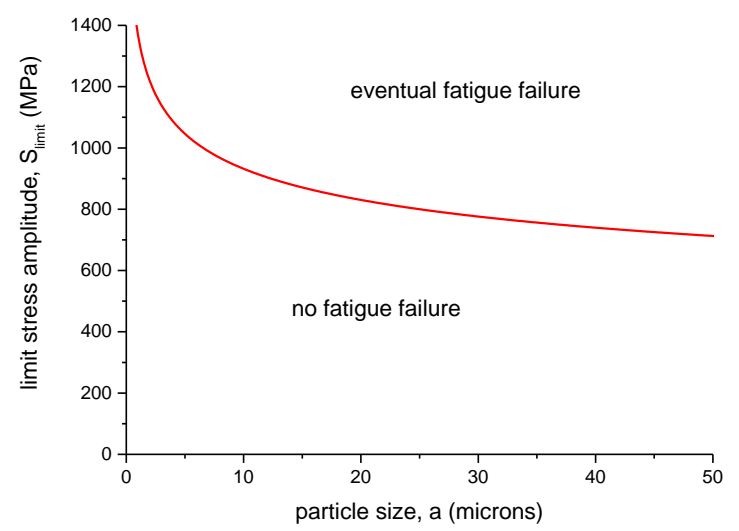

Figure 4: The fatigue limit stress (11) as a function of inclusion size. 


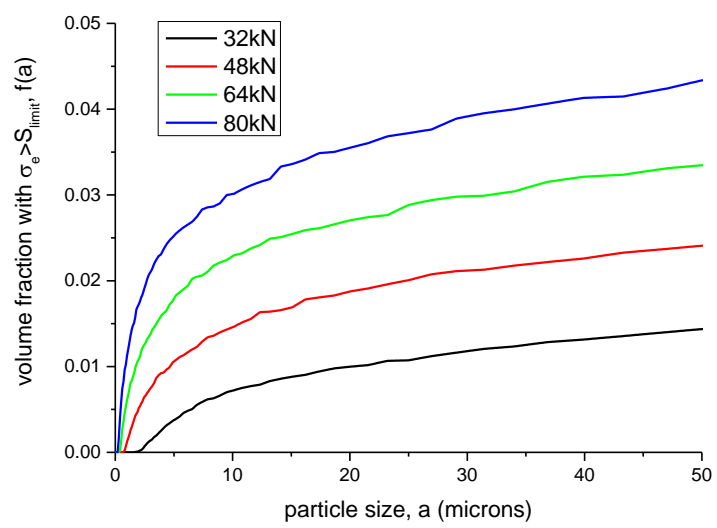

Figure 5: Combining Fig. $2 \mathrm{~b}$ and Fig. 4 gives the volume fraction of the raceway, $f(a)$, where the stress is large enough to initiate fatigue from inclusions of size $a$. 


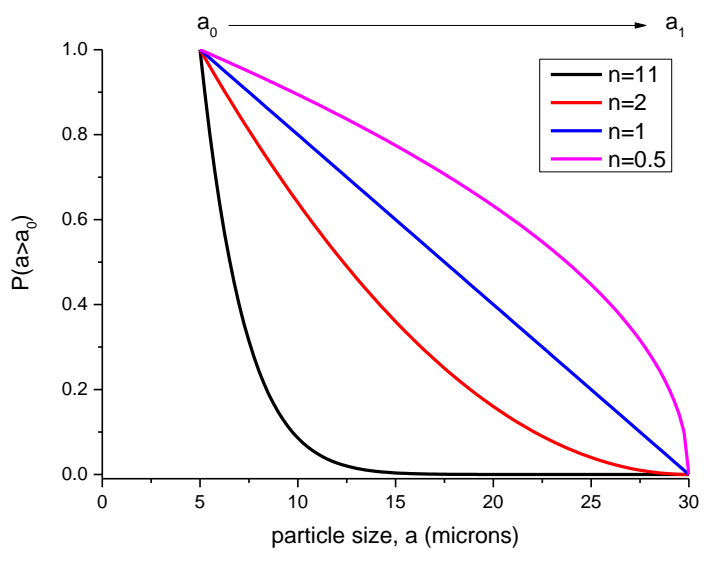

(a)

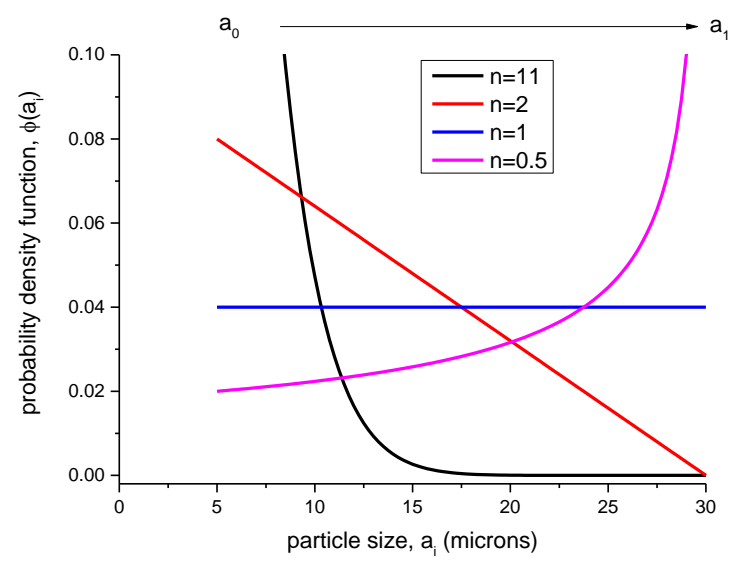

(b)

Figure 6: The (a) cumulative probability GPD function (12), and (b) its probability density function (13), for a threshold size of $a_{0}=5$ microns, maximum size $a_{1}=30$ microns, and various values of the exponent $n$. 


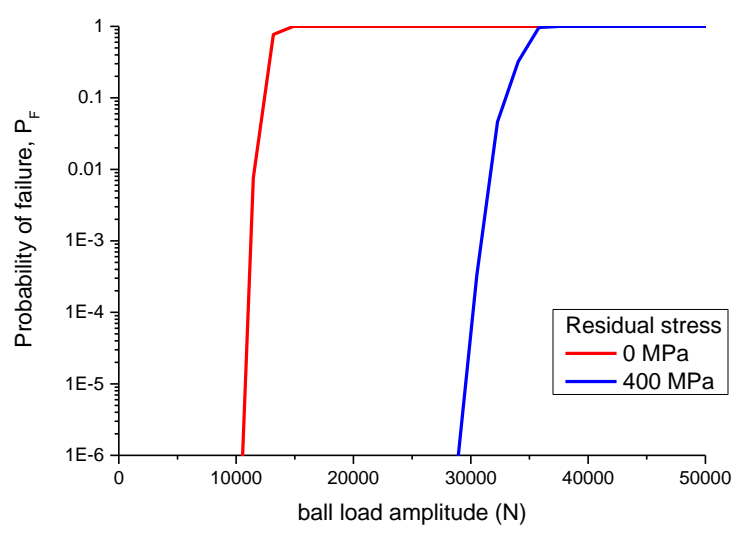

Figure 7: The failure probability for a sample with $a_{0}=5$ microns, $a_{1}=30$ microns, $n=11$, with a compressive residual stress field (400MPa) and without (0 MPa). 


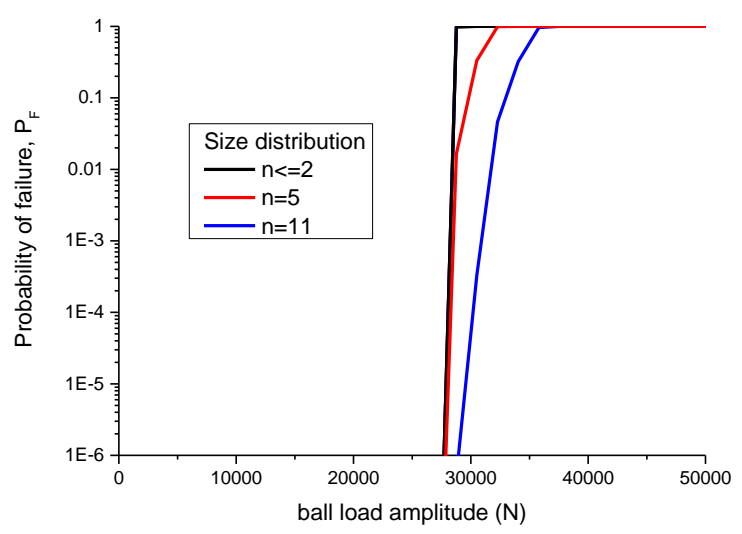

Figure 8: The failure probability for a sample with $a_{0}=5$ microns, $a_{1}=30$ microns, a compressive residual stress field (400MPa) for various values of $n$. 


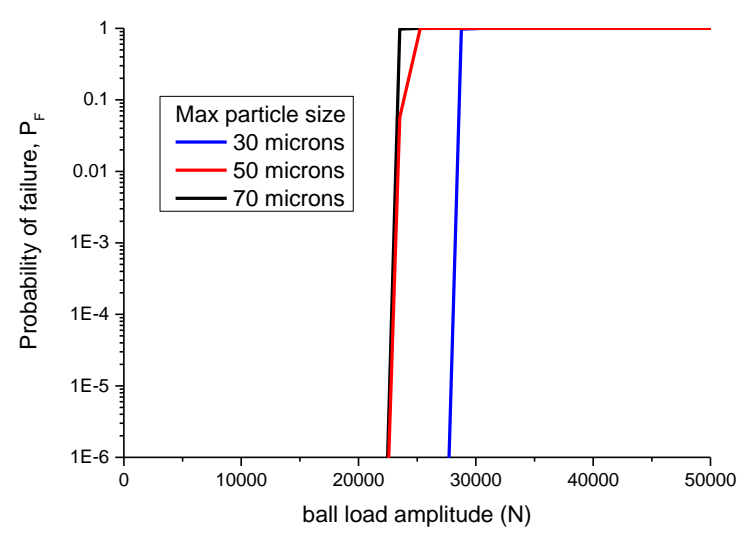

(a)

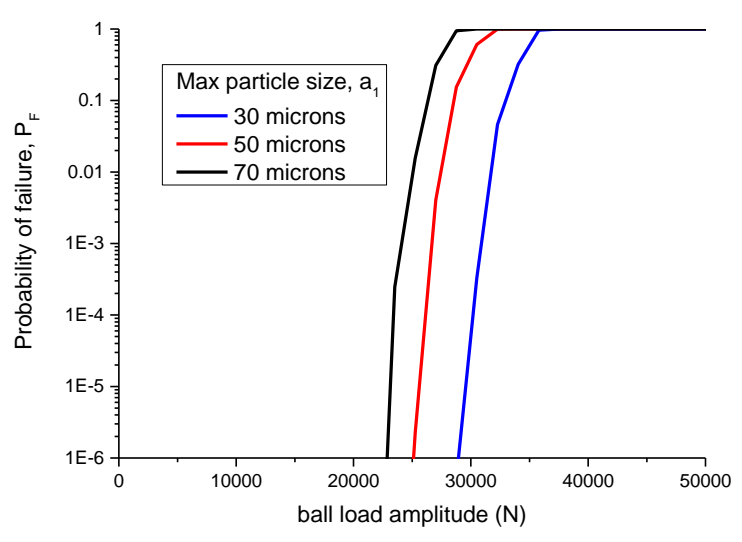

(b)

Figure 9: The failure probability for a sample with a compressive residual stress field (400MPa) with $a_{0}=5$ microns and values of $a_{1}=30,50$ and 70 microns for (a) $n=2$ and (b) $n=11$. 


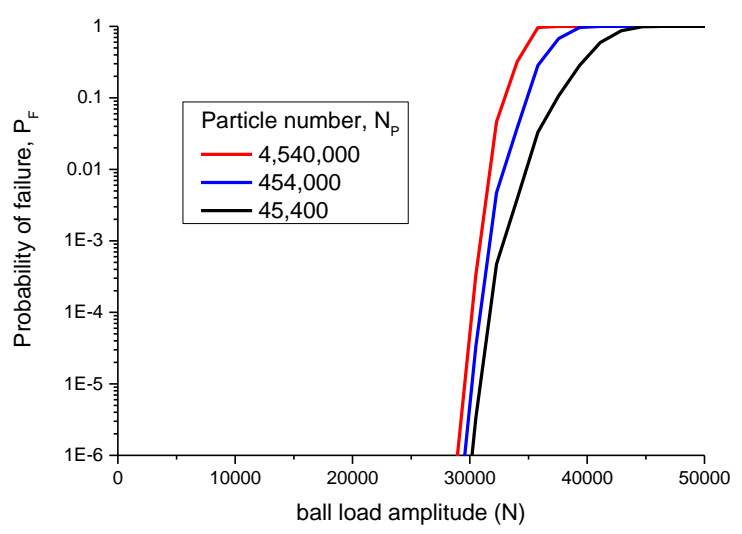

Figure 10: The failure probability for a sample with a compressive residual stress field (400MPa) with $a_{0}=5$ microns, $a_{1}=30$ microns, $n=11$ for different values for the number of inclusions in the raceway $N_{P}$. 